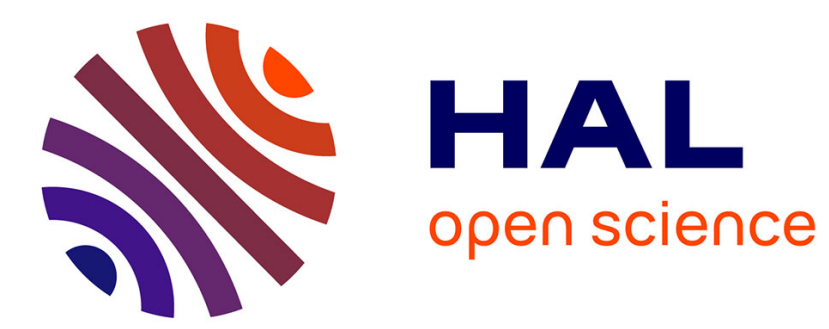

\title{
HICOFED OUTLOOK
}

\author{
M. Lefort
}

\section{To cite this version:}

M. Lefort. HICOFED OUTLOOK. International Conference On Heavy Ion Nuclear Collisions In The Fermi Energy Domain Hicofed 86, 1986, Caen, France. pp.C4-427-C4-434, 10.1051/jphyscol:1986451 . jpa-00225817

\section{HAL Id: jpa-00225817 https://hal.science/jpa-00225817}

Submitted on 1 Jan 1986

HAL is a multi-disciplinary open access archive for the deposit and dissemination of scientific research documents, whether they are published or not. The documents may come from teaching and research institutions in France or abroad, or from public or private research centers.
L'archive ouverte pluridisciplinaire HAL, est destinée au dépôt et à la diffusion de documents scientifiques de niveau recherche, publiés ou non, émanant des établissements d'enseignement et de recherche français ou étrangers, des laboratoires publics ou privés. 


\title{
HICOFED OUTLOOK
}

\author{
M. LEFORT
}

Institut de Physique Nucléaire, BP 1, F-91406 Orsay, France

\begin{abstract}
Since the subject of HICOFED is rather new, it would be certainly a mistake to draw any kind of conclusion from this lively meeting. Neither could we make a summary. Although I am not sure to well understand what it means, let us call "outlook" the few considerations which follow:
\end{abstract}

I should like to tell you about some aspects which appeared to me quite typical and rather exciting for the future. Probably these remarks will not be accepted by everybody, but this is at least the privilege of the last talk of the Conference, and probably the last talk in my scientific life, to be able to assert various considerations without being contradicted.

\section{GENERAL REMARKS}

The ma in topics of the Conference were selected one year ago and it seems to me that the choice was not so bad. Therefore, it may be worthwhile to follow it. Before, let us consider some general aspects. When the first experiments in the Fermi energy domain started with the CERN 85 MeV.A Carbon beam, we had nearly no idea about what we should look for. It was a little like the sailors who were landing 500 years ago on the shore of the new world and who were looking at the country wi th their european eyes and habits. So, we had to use rather simple concepts and methods that we borrowed from other domains of nuclear reaction studies, i.e. from the heavy ion collisions either at lower energies or at very high energies. Then, it was not surprising that the German group and the Scandinavian-Grenoble group were inspired by the abrasion model taken from the high energy side whereas we were, at Orsay, more interested in the linear momentum transfer and its connection with fusion process. Nowadays, it is really striking to observe that there are enough experimental data and theoretical approaches so that specific attempts can be devoted to the intermediate energy domain. The second remark is that, after a period of three or four years during which jnclusive measurements were carried on, equipments and ideas are ready for developing coincidence experiments and multiplicity measurements.

Comparing with the evolution of the studies in the low energy region during the seventies it is interesting to notice how the situation is different. The low energy - experiments started nearly without any theoretical model and the first results on what was called later on Deep Inelastic Collisions were totally unexpected, since only two classes of collisions were considered, compound nucleus formation and quasielastic transfer reactions. We were indeed delighted when my old friend Dieter Gross, around 1970, produced $a$ model which was able to describe our results ${ }^{2}$ by introducing friction forces along the deflection function of the collision. At that time, it was hard to follow a leading thread and to find a frame in order to make prospects for new experiments.

Nowadays, in the Fermi Energy Domain, the situation is very different, as it appears particulariy well in this Conference. The abundance of theoretical approaches is really impressive, and the difficulty is perhaps to decide which of them is the most useful for designing more specific experiments. It is one of the thoughts of Einstein that theory should be elaborated in the purpose of designing which observable should be searched. 
My last remark concerns the so called "exclusive" experiments. Nearly every talk was ended by the call for exclusive experiments. I am not sure that we are always prepared for them. Strictly speaking, do we know clearly what should be triggered and what should be excluded? There are a few cases where coincidence experiments have been carried on with success, because they were very specifically prepared by precise hypothesis made in the frame of inclusive results. Typically, it is the case of fast light particles emitted in coincidence with recoiling quasi-compound nuclei. Those particles travel with the beam velocity and their observation brings an evidence that the incomplete linear momentum transfer and incomplete fusion can be treated like an energy deposit from a partial mass of the projectile (see S. Leray's contribution). Another example is the study 3,4 of light charged particles emitted isotropically in the center of mass of the recoiling heavy nucleus source. It shows that a very excited heavy system evaporates protons, alpha particles, deuterons and probably neutrons prior to fission, even when the excitation enerạy per nucleon reaches more than $3 \mathrm{MeV} / \mathrm{n}$.

I am sure that the fragment multiplicity will be measured soon in various conditions, i.e., in peripheral and in central collisions, with the lowest possible energy threshold. Preliminary results were already given. My personal regret is that the delay is now so long between data acquisitions and the final result, at least for people like me who are eager to really understand what happens to these nuclei heated at $1 \mathrm{GeV}$ !

Let us now review briefly the varjous topics.

\section{NUCLEUS-NUCLEUS POTENTIAL - ELASTIC SCATTERING - TOTAL REACTION CROSS SECTION}

The heavy ion optical potential model is always the starting point for describing the scattering and the reactions. The extension of the Brückner method to heavy ions like it was described by $A$. Faessler seems to me quite an important progress. It is indeed also interesting to see how the sudden and the adiabatic optical potentials are nearly identical, both for the real part and the imaginary part, at the surface of the nucleus. Therefore, it justifies to treat the first step of the collision in terms of the sudden approximation (see fig. 6 in A. Faessler's contribution).

The knowledge of total reaction cross sections with a great accuracy is indeed quite important, particularly in the Fermi Energy Doma in where the nucleon mean free path in varying strongly, where the $\mathrm{N}-\mathrm{P}, \mathrm{N}-\mathrm{N}$ and $\mathrm{P}-\mathrm{P}$ cross sections are decreasing. Also we would like to know more about the neutron skin especially for light nuclei. The experimental methods, as they were described by Bruandet have been improved and it will be quite interesting to apply them for $\sigma_{R}$ measurements on reactions like

$12 c+16 c, 12 c+11 c$, where the neutron skin changes so much, when secundary "exotic" beams are available. We shall come back to this point later.

\section{DISCRETE STATE EXCITATION - QUASI-ELASTIC REACTIONS - GIANT RESONANCES}

The big spectrometer SPEG is now operating at GANIL. It is too early to know about its usefuiness. It is certainly the best tool for studying quasi-elastic transfer reactions which still occur at $50 \mathrm{MeV} . \mathrm{A}$, but with very low cross sections and in an angular range in the vicinity of zero degree. The transfer of one proton from $208 \mathrm{~Pb}$ to $209 \mathrm{Bi}$ induced by 160 at $50 \mathrm{MeV}$. A was observed with the effect of matching by population inversion. It is too early to say if the very high selectivity will be of such interest that it compensates the low cross section. Spin flip studies are also a rather new aspect of the high velocity of the projectile.

Moreover, let us insist a little more on the beautiful experimental confirmation of the structures in the inelastic scattering spectra which were observed at GANIL with the Argon beam as well as at SARA and Vicks $i$ with the Neon beam. Ph. Chomaz has shown us on $40 \mathrm{Ca}, 90 \mathrm{Zr}, 120 \mathrm{Sn}$ and $208 \mathrm{~Pb}$, together with a highly excited giant quadrupole resonance, structures ranging up to $60 \mathrm{MeV}$ excitation energy for the $40 \mathrm{Ar}$ inelastic scattering at $44 \mathrm{MeV} . \mathrm{A}$ (see the figure presented in his talk). The attribution to a strong multiphonon excitation seems a very convincing explanation. We understand now much better how this process can contribute to a fast energy dissipation at the early stage of the nucleus-nucleus collision. Also, one should consider how multi- 
phonon excitation is compatible with the Pauli principle in the frame of the sudden approximation. At lower energies, such a dissipation is followed by the deep inelastic process. For higher velocities, such an energy dissipation may be at the origine of the observed slowing down effects on projectile like fragments which have to be taken in account in peripheral collisions below $100 \mathrm{MeV}$.A.

\section{PERIPHERAL COLLISIONS - PROJECTILE-LIKE FRAGMENTS - EMISSION OF NUCLEONS}

Projectile-like fragments emitted with a velocity not very far from the initial projectile velocity have been studied for quite a few years. But also lighter fragments are emitted in a wide spectrum of quite lower energies. Moreover, there are tragments issued from a source travelling at a velocity close to half the beam velocity. That work started naturally with high energy considerations in mind, and the elegant Gol dhaber 5 prescription was followed since it was so successful at high energies. It is of course always possible to extract a width from a bell shape spectrum, even if the distribution is not symmetric. Therefore, $\sigma$ values were extracted, and compared with $\sigma_{0}$, the Fermi momentum width, according the popular expression :

$$
\sigma^{2}=\sigma_{0}^{2} \frac{A_{p}\left(A_{p}-A_{F}\right)}{A_{p}-1} \quad \text { and } \quad \sigma_{0}^{2}=\frac{\left\langle p_{F}^{2}\right\rangle}{5}
$$

where $A_{F}$ and $A_{p}$ are the masses of the observed fragment and of the projectile respectively (see for example R. Dayras' contribution).

It is now rather clear that such a procedure may lead to wrong conclusions when applied to the Fermi Energy Doma in. What we observe there is not the "fragmentation" described at 1. GeV.A. First, there is a contribution of classical transfer reactions which should be subtracted even for fragments with $Z$ values equal to $Z_{p}-2$. Second, as was already mentioned,slowing down processes due to the/mean field are still occuring, even if the clutching into a two center intermediatexis not produced. Third the separation into spectators and a participant which is the basis of the abrasion-fragmentation model, is indeed too schematic. Particularly it is difficult to imagine a clean-cut of the projectile spectator and a participant zone flying away like the "fireball" and a target spectator.

A big effort has been made in several laboratories to build-up large multidetectors and coincidence experiments have started for measuring both fragments and fast light charged particles. At GANIL the huge chamber "Nautilus" has been used in that purpose and we have heard the first resuits on the system $40 \mathrm{Ar}+\mathrm{Ag}$ at $35 \mathrm{MeV}$. A. It is rather surprising, for example, to see that $50 \%$ of the fragments $Z=16$ are emitted without being escorted by a light fragment. But we don't know if the primary fragment was $Z=18$ and because of $i$ ts excitation energy evaporated an alpha particle (not observed by the plastic wall of Nautilus), or if two protons or an 4he were directly transferred to the target. So, we may repeat that, before making very exclusive experiments, one should accumulate more information about multiplicities and various sets of coincidence experiments.

Particle-particle correlations have been made. Their purpose is to try to detect a hot zone from which the fast emission would originate. There are many objections against this interpretation and for the moment the situation is rather confused. Amongst the coincidence experiments between the quasi-projectile and light charged particles, it is worthwhile to notice an interesting attempt to observe the defiection of the particles by the nuclear mean field, using for that the amount of circular polarisation of $\gamma$ rays in coincidence. The result indicates that they are emitted predominantly to negative angles, which is consistent with the deflection by an attractive nuclear mean field. If the interaction was purely of the type nucleon-nucleon collision, the polarisation should be smaller. Perhaps we are here in presence of a new method, typically adapted to the Fermi energy domain, which is able to estimate the interplay between nuclear mean-field and nucleon-nucleon collisions (see communication 27 by M.B. Tsang).

In the frame of what was noticed in the introduction, specific theoretical models are appearing and were presented at this Conference, which describe dissipative collisions 
in the intermediate energy range by treating both mean-field and nucleon-nucleon interactions. There are several approaches, all refering more or less to the BoltzmannUehi ing-Uhlenbeck equation. In that respect, the movy picture presented by Grégoire was really very suggestive. On the other hand such treatments look a little like black magic boxes for experimentalists and it is sometimes nice to follow rather simpler approaches like the production of fragments as a result of collisions of projectile nucleons with target nucleons (see B. Harvey). The neutron enrichment of the fragments which is observed when the target is neutron rich would then be the result of the known fact that $n-p$ scattering cross section is about three times larger than $n-n$ or $p-p$ cross sections, in the range of kinetic energy which is of interest for us.

A last trivial remark : we are still unable to answer precisely to the question : What is the energy, or the velocity, where Deep inelastic collisions are not seen any more ? This very simple question is related to the important problem to know when the clutching becomes so difficult that it fails. It is also related to the possibility of fusion for a given partial wave. A very naive idea is to compare the average relative velocity of projectile nucleons, $\bar{v}_{p}$, with the Fermi velocity $v F$. The average kinetic energy of incident nucleons is $\left\langle\mathrm{E}_{k}\right\rangle=\mathrm{m}_{0} / 2\left(\bar{v}_{\mathrm{p}}+3 / 5 \mathrm{vF}\right)^{2}$ and the clutching becomes difficult if $\left\langle E_{k}\right\rangle \geq E_{F}+S$, where $S$ is the average separation energy of a nucleon. This would correspond to $\vec{v}_{p}$ around $0.2 \mathrm{c}, \mathrm{i} . \mathrm{e}$. around $20 \mathrm{MeV} / \mathrm{n}$. Rudolf has shown us the very typical deep inelastic resuit in the reaction 92 Mo +92 o at 18.2 MeV.A, with a huge energy dissipation, whereas for $86 \mathrm{Kr}+98 \mathrm{Mo}$ and $86 \mathrm{Kr}+93 \mathrm{Nb}$ at $35 \mathrm{MeV} . A$, other processes, quite new, are replacing D.I.C.

\section{CENTRAL COLLISIONS - HIGHLY EXCITED NUCLEI - EQUATION OF STATE AND PHASE TRANSITION}

It is not so clear to define a central collision when, for example one realises that at $30 \mathrm{MeV} . \mathrm{A}$, a Krypton projectile carries a linear momentum of $19 \mathrm{GeV} / \mathrm{c}$, but a.l so can induce an angular momentum of $100 \hbar$ for an impact parameter as smali as 1 Fermi.

In Central Collisions Studies there are three main aspects: linear momentum transfer (LMT) related to the extent of fusion, highly excited nuclei, limit in temperature or excitation energy for a nuclear system.

In order to know to which extent the fusion process between two nuclei is complete or not, the estimate of the linear momentum transfer is an obvious method. The measurements are made by determining velocities and possibly masses of recoiling nuclei. The result that fuT1 momentum transfer could not be observed 6 any more with $12 \mathrm{C}$, at $30 \mathrm{MeV} . \mathrm{A}$ was the beginning of a series of studies. The data are often presented in terms of $\tilde{\rho}$, which is the ratio of the average LMT to the initial 1 inear momentum (so called Viola systematics7). Sylvie Leray has shown us a simple relationship between $\tilde{\rho}$ and the relative velocity $\left(\left(E-V_{C}\right) / A\right) 1 / 2$. There are theoretical treatments, using Boltzmann equation which reproduce more or iess this behaviour, assuming that nucleon-nucleon interactions inhibit more and more the mean-field fusion process when the velocity increases.

But another presentation of the results works as well. It was suggested by SaintLaurent et al on the basis of a systematic study of helium induced reactions 8 and then was generalized to heavy ions 9 . The essential remark is that the limit increases with the mass of the projectile and finally corresponds to a value of the order of $180 \mathrm{MeV} / \mathrm{c}$ per nucleon. Such a remark directs towards other possibilities for explaining the result. For example one may notice that the corresponding velocity, $0.2 \mathrm{c}$, approaches the estimate of the sound velocity in nuclear matter.

\section{Fusion, Incomplete fusion, Energy deposit}

The relation between linear momentum and energy deposition is straight forward if the lack of momentum is due to a lack of transferred mass, as this has been demonstrated. Therefore nowadays, we know that excitation energies close to $1 \mathrm{GeV}$ can really be deposited in nuclear composite systems, as I made the hypothesis 9 already at the German Physical Society in Bad Honeff (1981) after the CERN experiments. The simple relation is the following :

$$
E^{*}=\frac{\langle p\rangle^{2}}{2\left\langle M_{p}^{\top}\right\rangle} \cdot \frac{M_{T}}{\left\langle M_{p}\right\rangle+M_{T}}+Q
$$


where $\langle p\rangle$ is the mean transfer of linear momentum, $M_{p}^{\prime}$ the mass incorporated into the fusion to the target mass $M T$, assuming $\langle p\rangle / p_{i}=\left\langle M_{p}\right\rangle M_{p}$. Jacob Bondorf told me that we are discovering what Niels Bohr was telling in 1936, exactly 50 years ago, in one of his three papers where the compound nucleus was invented : "It is conceivable that a compound nucleus may sustain an excitation energy of the order of $1 \mathrm{GeV}$ ".

But a set of experiments have shown that the statistical treatment is not entirely correct when it predicts for very heavy systems the predominance of fission over any particle evaporation. We heard at this meeting that a more elaborated analysis applying the Fokker-Planck equation to the very short-lived composite system predicts indeed to a large extent light charged particle and neutron emission prior to fission. H. Delagrange has shown that when fission does occur, nuclei have lost indeed so many neutrons that they are not so hot. Also, if the composite system has been so much depleted in mass, the fission process may not occur at al1. This was experimentally shown in the case of $\mathrm{Ar}+165 \mathrm{Ho}$ by Rivet et a1. (communication 39).

A careful analysis of the kinetic energy spectra of light charged particles emitted in backward angles (the evaporation origin should be checked) is probably the only way to estimate the temperature of such excited nuclei. There are several cares that should be taken. J. Alexander has explained how the angular momentum and the deformation could modify both the slope and the maximum of the spectra. Also nuclei are cooled down through a long cascade of particle evaporation and as Natowitz has pointed out, the experimental result is an averaging of a long set of decreasing temperatures from the very hot initial system down to a nearly. cold nucleus. Fortunately, for very heavy systems, a small number of 1 ight charged particles (alpha and protons) are evaporated in the very first stage, followed by neutron emission and finally fission. Therefore a natural selection is made of very hot species 4 . A last word about the important improvement due to the measurements of neutron multiplicities which are now possible with the help of the "old" neutron scintillator ball. It provides a very direct tool to estimate the excitation energy and at the same time the reaction cross section (see Jahnke's contribution).

Now we are overcoming the limits of $E^{\star}$ above which the known processes of de-excitation are not observed any more. I am more and more convinced of that experimental fact, but we still don't know what is its signification. In that respect the recent systematic study at various incident energies for the angular correlation of fission fragments emitted in the reaction $58 \mathrm{Ni}+232 \mathrm{Th}$ shows very typically what was al ready observed for $40 \mathrm{Ar}+238 \mathrm{U}$, i.e. the decrease in height for the bump of large momentum transfer at $9.5 \mathrm{GeV} / \mathrm{c}$ (C. Volant, communication 40). The corresponding excitation energy for the highest bombarding energy of $30 \mathrm{MeV} . \mathrm{A}$, reaches $920 \mathrm{MeV}$, about the same as for Ar $+U$ at $35 \mathrm{MeV} . \mathrm{A}$. This is just on the shore of the 1 imit around $\varepsilon^{*}=E^{*} / A=3.5 \mathrm{MeV}$ per nucleon. Then the figure which was given 10 last year is still valid. It has been confirmed by some new results which I have added.

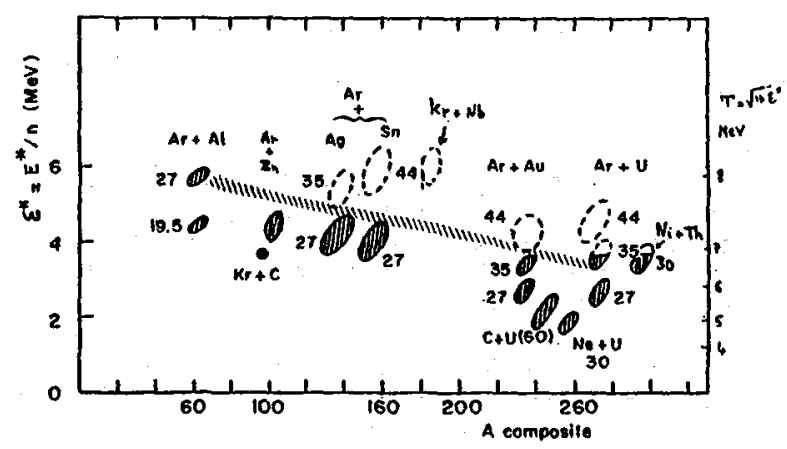

Fig. 1 - Excitation energy per nucleon deduced from various observations of i inear momentum transfer and corresponding residual nuclei or fission fragments. The spots cover complete and incomplete fusion with linear momentum transfer ranging from $\langle p\rangle / p=1$ down to $\langle p\rangle / p=0,6$. Full spots are for the case where fusion occurs. Dashed spots are for those where the cross section is less "than $200 \mathrm{mb}$. The zone where seems to occur a critical limit is shown. 
What happens ? What should we look for ? Here is a real challenge. A very attractive possibility is the multifragmentation which was proposed by $\mathrm{J}$. Bondorf in the frame of thermal statistical theory, and by $X$. Campi using a percolation model. Both approaches lead to similar conclusions and are physically equivalent. Furthermore they could concern central collisions as well as those collisions with larger impact parameters "for which one observes light fragments originating from a source at roughly half the beam velocity (Borderie). There are also the interesting results given by Rudolf on two fragment correlations for collisions between Krypton + Molybenum and Xenon + Silver. But the real answer will be given by carefull multiplicity measurements of light and medium mass fragments, and for the moment we have in hands only the results of nuclear emulsions, which are indeed good $4 \pi$ detectors. The experimental effort to be made for electronic counters is huge and we have unfortunately to wait.

Now, of course, multifragmentation is not the only theoretical possibitity which has been proposed, and we heard how a hot nucleus could undergo vaporisation at a temperature higher than some $8 \mathrm{MeV}$, in the frame of a static + dynamic treatment of the nuclear interaction with the mean-field.

Finally, the proposal of equation of state and phase transition in nuclear matter, which was made several years ago, has lead to many theoretical discussions more than to a real prediction for a crucial experiment.

\section{COOPERATIVE PROCESSES}

Only a very few words on the subject. The introduction of highly energetic gamma rays as a possible effect of cooperative processes in the nuclear collisions at energies between $30 \mathrm{MeV}$.A and $100 \mathrm{MeV}$. A has been very fruitful. The idea of proton-neutron bremsstrahlung 11 . goes back to 1966, and the results shown by Pinston are quite convincing. The review on pion production made by Knoll and Gross is in favor of a cooperative model with composite particles as final states.

\section{PRODUCTION OF NUCLEI FAR OFF THE BETA STABILITY LINE}

The last but not the least : I should confess that I was not very optimistic about the interest of heavy ion projectiles as compared to energetic protons for the production of new isotopes, particularly along the drip line. The reason was that I was thinking only about the evaporation residues. As D. Guerreau has shown us, the peripheral reactions are indeed a very efficient tool which sprinkles both sides of the stability valley. The drip line has been reached for neutron rich nuclei up to $Z=20$. On the other side, interesting nuclei have been discovered 1 ike $31 \mathrm{Ar}$ which is a good candidatefor 2 proton radioactivity. Moreover spectroscopic studies have been carried on with very ingenious technics.

My main remark on this subject is finally on the experimental aspect. Most of the results indicated above were obtained because there was ready at GANIL since the beginning an apparatus called Lise, particularly well adapted for the identification of peripheral collision products. The construction of Lise was a difficult decision and I am proud that it was taken in time.

I should like to finish this paragraph with a remark on the secondary beams which were described by René Bimbot. Again, Lise appears like a wonderful instrument, since it is possible to select unstable reaction products, 1ike $17 \mathrm{~N}, 16 \mathrm{C}$ or $38 \mathrm{~S}$, to steer the beam of these nuclei and to reach a high degree of purification with an intensity which is already of interest, although we are only at the beginning of the technical improvements. Therefore, we have the hope that quite exotic beams will be produced at intensities of the order of $10^{8}$ particles per second and I am sure that this will open quite promising new set of experiments on reaction mechanisms as well as search for more "exotic" isotopes, since we shall handle wide varieties of isotopes both in the target and in the beam. 
Our HICOFED Conference comes to its end, three years only after the first beams at Vicksi, GANIL, SARA and MSU. You will forgive me to show aga in a figure where the ma in characteristics of our Energy Doma in are indicated (Fig. 2).

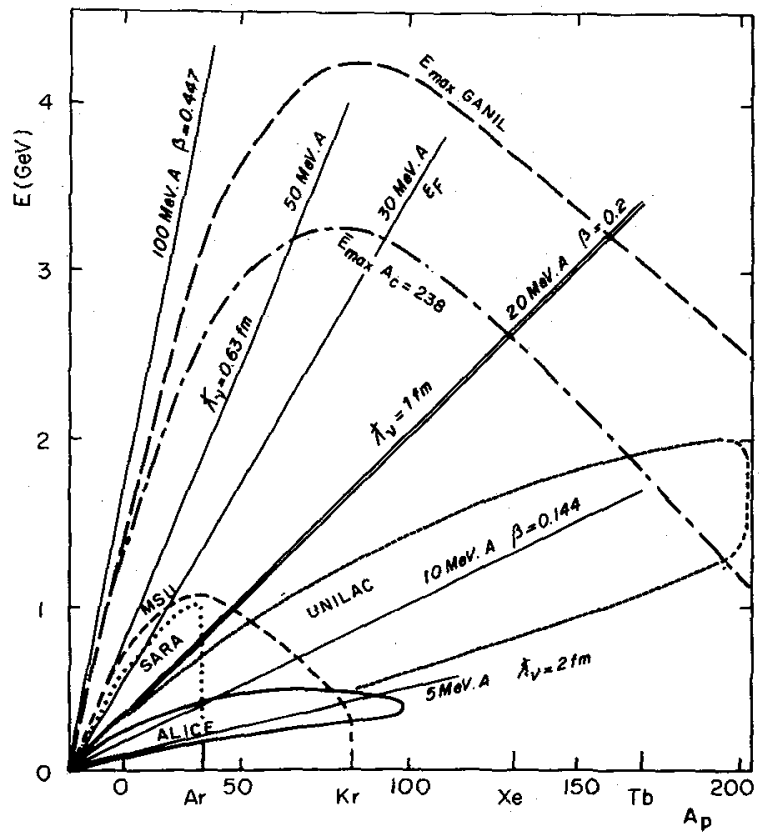

Fig. 2 - Energies in MeV.A, center of mass energies on $238 \mathrm{U}$ for various projectiles $A_{p}$. Also indicated nucleon wave length, velocities in $c$ units.

There were, along these five days, so many interesting results presented, and the discussion was so active that we can already claim that the choice of theses accelerators was indeed a good one. Also, both the theoretical ideas and the experimental equipment, which has been built and which is in preparation, give us the greatest hope for the future. I wish that the beautiful work which is going on will overcome the pessimistic propency which floats over nuclear physics particularly in our country. My last wishes will be for our colleagues who are expecting the success of their new accelerators in Catania, Chalk River, Texas, Tokyo and Lanchow. I hope that you will come back to your laboratories with in mind this Epictete's thought : "Those things belong to you that you have stored in your memory and that you keep in your heart".

\section{References}

1. Gross, D.E., Kalinowski, H., Phys. Lett. 48B (1974) 302.

2. Galin,J., Gatty, B., Lefort, M., Péter, J., Tarrago, $X_{.}$and Basile, R., Phys. Rev. 182 (1969) 1267.

3. Song, S., Rivet, M.F., Bimbot, R., Borderie, B., Forest, I., Galin, J., Gardès, D. Gatty, B., Lefort, M., Deschler, H., Tama in, B., Tarrago, X., Phys.Lett.130B (1983) 14.

4. Jacquet, D., Duek, E., Alexander, J.M., Borderie, B., Galin, J., Gardès, D., Guerreau, D., Lefort, M., Monnet, F., R.ivet, M.F., Tarrago, X., Phys.Rev.Lett. 53 (1984) 2226 .

Jacquet, D., Galin, J., Borderie, B., Gardès, D., Guerreau, D., Lefort, M., Monnet, F., Rivet, M.F., Tarrago, X., Duek, E., Al exander, J.M., Phys.Rev. C32 (1985) 1594 . 
5. Goldhaber, A.S., Phys.Rev. C17 (1978) 2243.

6. Bimbot, R., Borderie, B., Forest, I., Galin, J., Gardès, D., Gatty, B., Guillemot, H., Lefort, M., Oeschler, H., Rivet, M.F., Song, S., Tama in, B., Tarrago, X., Lecture Notes in Physics 158 (1982) 341.

Gal in, J., Deschler, H., Song, S., Borderie, B., Rivet, M.F., Forest, I., Bimbot, R., Gardès, D., Gatty, B., Guillemot, H., Lefort, M., Tama in, B., Tarrago, X., Phys.Rev.Lett. 48 (1982) 1787.

7. Back, B.B., Wolf, K.L., Mignery, A.C., Gelbke, C.K., Awes, T.C., Breuer, H., Viola Jr., V.E. and Dyer, P., Phys.Rev. C22 (1980) 1927.

8. Saint Laurent, F., Conjeaud, H., Dayras, E., Harar, S., Deschler, H. and Volant, C., Phys.Lett'. 110B (1982) 372.

9. Lefort, M., Nucl. Phys, A387 (1982) 3c-24c.

10.Lefort, M., Proc. Italian Phys. Soc., R.A. Ricci and C. Villi, 467 (1986). Borderie, B., IPNO-DRE-85-23 (1985).

11.Edgington, J.A., and Rose, B., Nuct.Phys. 89 (1966) 523. 